\title{
Mechanism of Multiple Grating Formation in High-Energy Recording of Holographic Sensors
}

\author{
Ali K. Yetisen \\ Harvard Medical School and Wellman Center for Photomedicine, AYETISEN@mgh.harvard.edu \\ Yunuen Montelongo \\ University of Cambridge \\ Nicholas M. Farandos \\ Imperial College London
}

See next page for additional authors

Follow this and additional works at: https://arrow.tudublin.ie/scschphyart

Part of the Optics Commons

\section{Recommended Citation}

Ali K. Yetisen,Yunuen Montelongo, Nicholas M. Farandos, Izabela Naydenova, Christopher R. Lowe, Seok Hyun Yun, (2014) Mechanism of multiple grating formation in high-energy recording of holographic sensors", Applied Physics Letters 105, 261106 (2014). doi:10.1063/1.4905352

This Article is brought to you for free and open access by the School of Physics \& Clinical \& Optometric Science at ARROW@TU Dublin. It has been accepted for inclusion in Articles by an authorized administrator of ARROW@TU Dublin. For more information, please contact arrow.admin@tudublin.ie, aisling.coyne@tudublin.ie, gerard.connolly@tudublin.ie.

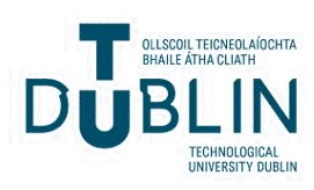




\section{Authors}

Ali K. Yetisen, Yunuen Montelongo, Nicholas M. Farandos, Izabela Naydenova, Christopher R. Lowe, and Seok Hyun Yun 


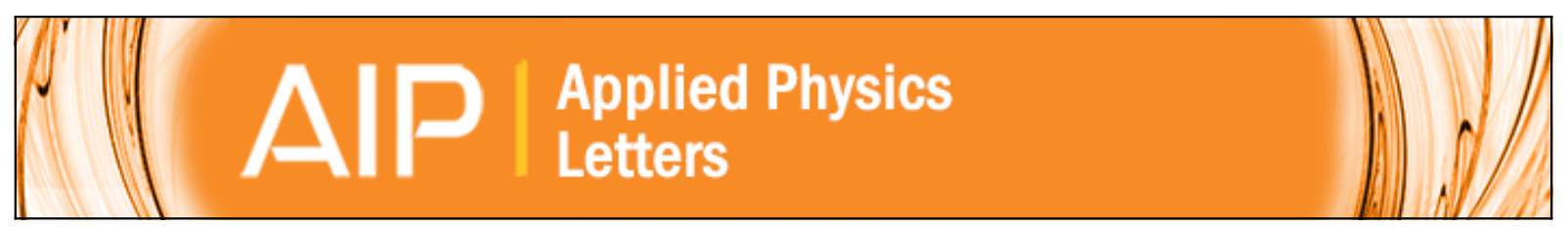

\section{Mechanism of multiple grating formation in high-energy recording of holographic sensors}

Ali K. Yetisen, Yunuen Montelongo, Nicholas M. Farandos, Izabela Naydenova, Christopher R. Lowe, and Seok Hyun Yun

Citation: Applied Physics Letters 105, 261106 (2014); doi: 10.1063/1.4905352

View online: http://dx.doi.org/10.1063/1.4905352

View Table of Contents: http://scitation.aip.org/content/aip/journal/apl/105/26?ver=pdfcov

Published by the AIP Publishing

\section{Articles you may be interested in}

Dynamic characterizations of high diffraction efficiency in volume Bragg grating formed by holographic photopolymerization

J. Appl. Phys. 106, 083111 (2009); 10.1063/1.3251413

Holographic inscription of helical wavefronts in a liquid crystal polarization grating

Appl. Phys. Lett. 91, 141112 (2007); 10.1063/1.2793173

Holographically formed Bragg reflection gratings recorded in polymer-dispersed Liquid crystal cells using a HeNe laser

Appl. Phys. Lett. 88, 051121 (2006); 10.1063/1.2168707

Mechanical tuning of holographic polymer-dispersed liquid crystal reflection gratings

Appl. Phys. Lett. 85, 1949 (2004); 10.1063/1.1790601

Polymeric wavelength filters fabricated using holographic surface relief gratings on azobenzene-containing polymer films

Appl. Phys. Lett. 82, 3823 (2003); 10.1063/1.1579847

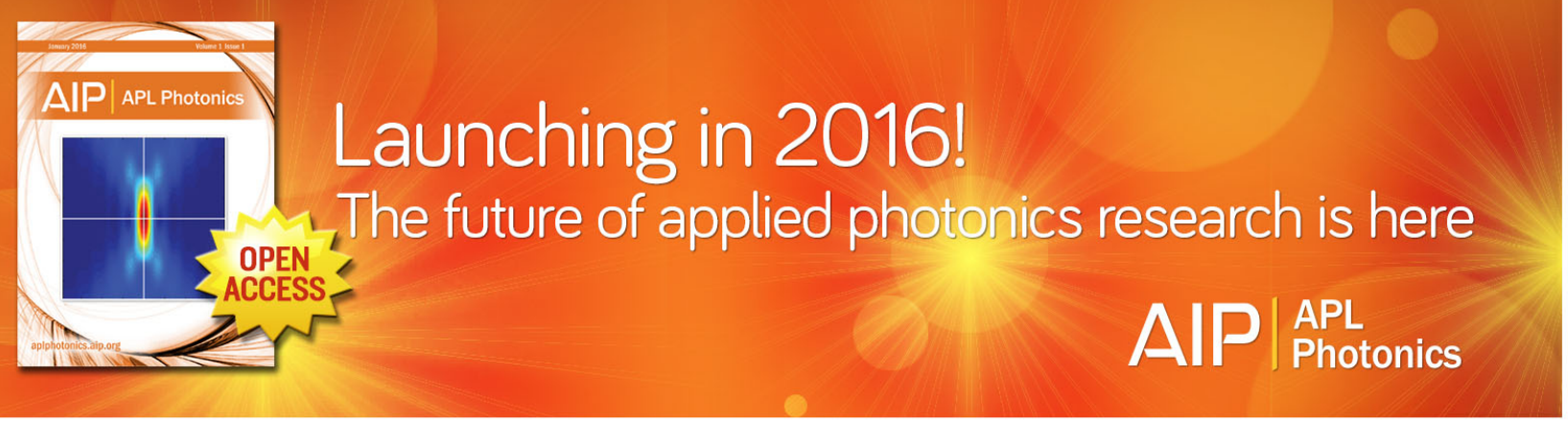




\title{
Mechanism of multiple grating formation in high-energy recording of holographic sensors
}

\author{
Ali K. Yetisen, ${ }^{1, a), b)}$ Yunuen Montelongo, ${ }^{2, b)}$ Nicholas M. Farandos, ${ }^{3}$ Izabela Naydenova, ${ }^{4}$ \\ Christopher R. Lowe, ${ }^{5}$ and Seok Hyun Yun ${ }^{1}$ \\ ${ }^{1}$ Harvard Medical School and Wellman Center for Photomedicine, Massachusetts General Hospital, Boston, \\ Massachusetts 02114, USA \\ ${ }^{2}$ Electrical Engineering Division, Department of Engineering, University of Cambridge, Cambridge CB3 OFA, \\ United Kingdom \\ ${ }^{3}$ Department of Chemical Engineering, Imperial College London, South Kensington Campus, London SW7 \\ 2AZ, United Kingdom \\ ${ }^{4}$ Centre for Industrial and Engineering Optics, School of Physics, College of Sciences and Health, Dublin \\ Institute of Technology, Dublin 8, Ireland \\ ${ }^{5}$ Department of Chemical Engineering and Biotechnology, University of Cambridge, Tennis Court Road, \\ Cambridge CB2 1QT, United Kingdom
}

(Received 27 November 2014; accepted 20 December 2014; published online 31 December 2014)

\begin{abstract}
We report numerical analyses of Bragg diffraction by Denisyuk reflection holograms recorded by a high-energy pulsed laser. An intensity threshold must be passed to pattern a multilayer reflection and transmission hologram, which exhibits a nonlinear fringe structure. Numerical evaluations are provided for the laser light intensity, readout diffraction offset angle, transmission of the layer, and thickness of the polymer matrix during hologram recording. A non-sinusoidal surface pattern is formed at the top of the multilayer structure, and its effect on the diffraction properties of the structure becomes significant when the recording tilt angle is increased. Experimental results show that the angle of the diffracted light increases nonlinearly according to the tilt geometry in grating formation. (c) 2014 AIP Publishing LLC. [http://dx.doi.org/10.1063/1.4905352]
\end{abstract}

Holography is a well-established 3D imaging technique that has applications in displays, ${ }^{1-4}$ data storage, ${ }^{5}$ metamaterials, ${ }^{6}$ nanofabrication, ${ }^{7}$ imaging, ${ }^{8}$ non-destructive testing, ${ }^{9}$ interferometry, ${ }^{10}$ optical elements for spectroscopy, ${ }^{11}$ laser beam combination, ${ }^{12}$ and telecommunication. ${ }^{13}$ These devices can operate by modulating the properties of the diffraction gratings by single or multiple laser-induced interferences. Holography also represents a practical approach to fabricate optical sensors for applications in the quantification of chemical, biological, and physical stimuli. Holographic sensors incorporate gratings within stimuli-responsive hydrogels to modulate the Bragg diffraction. ${ }^{14}$ Laser-induced fabrication of the sensors involves multi-beam interference-based photochemical patterning. The major advantages of the holographic sensors over other optical sensors are the capability to produce 3D images, control over the angle of off-axis diffraction, and amenability to mass manufacturing. These sensors can quantify the concentrations of metal ions, ${ }^{15}$ glucose, ${ }^{16}$ and hormones ${ }^{17}$ in aqueous solutions. This technology also represents a platform, which can be integrated with microfluidic devices, ${ }^{18}$ contact lenses, ${ }^{19}$ and smartphone readers. ${ }^{20}$

Holographic sensors have traditionally been fabricated by photochemistry. This method requires dyes to photosensitize recording media by forming silver halides before writing a latent image. ${ }^{21}$ The image is generated using multi-beam interference under safelight and amplified using a photographic developer to produce a narrow-band Bragg diffraction grating. However, silver halide chemistry is limited in

\footnotetext{
a) Author to whom correspondence should be addressed. Electronic mail: ay283@cam.ac.uk.

${ }^{b}$ A. K. Yetisen and Y. Montelongo contributed equally to this work.
}

fabricating sensors because photosensitizing dyes are toxic for enzymatic assays, cause undesired light absorbance, and require time-consuming chemical processing steps. ${ }^{22}$ Recently, high-energy pulsed laser recording has been proposed for the production of holographic sensors in hydrogel matrices. ${ }^{23}$ The mechanism of grating formation in pulsed laser recording has been attributed to change in nanoparticle (NP) size, displacement by means of thermal energy, and metal oxide shell formation. ${ }^{23-25}$ The physical parameters such as incoming wavelength, power, pulse duration, nanoparticle size, and surface plasmon resonance influence the optical characteristics of photochemical patterning. ${ }^{26-28}$ When holograms are produced in Denisyuk reflection mode, in addition to the desired multilayer grating running parallel with the plane of the polymer film, a transmission grating running almost perpendicular to the plane of the film tends to form due to the reflection from the lower surface boundary of the emulsion and air. ${ }^{16,23,29}$ For optimal operation of a holographic sensor comprising the multigrating photonic structure, it is important to minimize the formation of transmission grating while maximizing the monochromatic Denisyuk grating. The analytical solution for image recording through silver halide chemistry has been described by Kogelnik theory. ${ }^{30}$ The coupled wave theory allowed understanding diffraction in sinusoidal volume gratings and provided analytical formulas for the computation of diffraction efficiency. Another approach to Kogelnik's theory is parallel stacked mirrors model, which is based on the differential formulation of the Fresnel reflection within the grating. ${ }^{31}$ However, no systematic approach has been developed to understand the fundamentals of the pulsed laser induced 
formation of multilayer and transmission gratings. In addition to the geometric effect, understanding the mechanism of nanosecond light interference with pulsed lasers and subsequent image recording will allow improving fabrication methods.

Here, we analyze the formation of Denisyuk holograms and associated surface transmission gratings by high-energy pulsed lasers. We evaluate the diffraction characteristics of devices computationally for various tilt angles, threshold optical intensities, absorption values, and thickness of hydrogel matrix. We then fabricate holograms at different geometries by using a high-energy nanosecond pulsed laser and characterize their optical properties by reflection spectrophotometry and imaging. The results provide mechanistic insights into the fundamental process of recording and reading and demonstrate key parameters for the design and fabrication of holographic sensors.

In the computational model, we considered a planewave writing beam propagating from a $\mathrm{Nd}$ :YAG pulsed laser $(\lambda=532 \mathrm{~nm}, 6 \mathrm{~ns}$, and $350 \mathrm{~mJ})$. The interference patterns in Denisyuk reflection mode were determined by the superposition of three main waves: incident beam $\left(\lambda_{1}\right)$, beam reflected from the mirror $\left(\lambda_{2}\right)$, and beam reflected internally at the hydrogel-water interface $\left(\lambda_{3}\right)$ (Fig. 1). The recording medium was a $10-\mu \mathrm{m}$ thin layer of poly(2-hydroxyethyl methacrylate) (pHEMA) matrix doped with $\mathrm{Ag}^{0}$ NPs. Based on the effective refractive indexes and attenuation coefficients of different layers, we computed the respective intensities and phases of individual plane waves and determined the resulting interference patterns in the recording medium $\left(10 \times 10 \mu \mathrm{m}^{2}\right)$. To simulate the laser-induced patterning, it was assumed that the energy of a single pulse was transmitted instantaneously to the medium before heat diffusion was involved. $^{29}$

A threshold function was defined as the patterned regions and allowed producing the multilayer structure of the grating. This threshold was established at a specific intensity of the field retrieved. The interference produced by the propagated field was obtained by mathematically defining three complex oscillations. With the complex representation

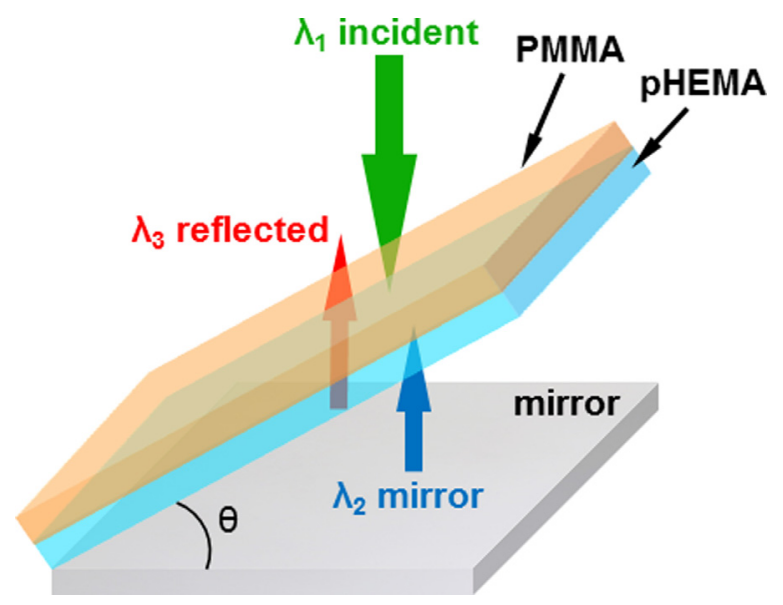

FIG. 1. Schematic of the patterning for the formation of holograms in Denisyuk reflection mode, showing the interference pattern of three waves: an incident beam $\left(\lambda_{1}\right)$, a beam reflected from mirror $\left(\lambda_{2}\right)$, and a beam reflected internally at the hydrogel-water interface $\left(\lambda_{3}\right)$. of the field, the interference profile was computed by adding the waves. Furthermore, the exponential decay produced by the absorption of the recording medium creates a variation along the direction of propagation of the beam. Figure 2(a) illustrates a typical normalized intensity field distribution obtained from a recording medium with varying tilt angles from $0^{\circ}$ to $20^{\circ}$ while the transmission $(20 \%)$ and thickness $(10 \mu \mathrm{m})$ were kept constant. The incident beam was propagated from the top in Denisyuk reflection mode, as evident from the intensity of the wave, which exponentially decayed as it propagated through the recording media. Although Fig. 2(a) shows the intensity distribution, the holographic structure formation occurs at a threshold. Therefore, only the waves with above certain intensities lead to patterning. When the threshold was applied to the simulations shown in Fig. 2(a), multilayer patterns were obtained (Fig. 2(b)). The threshold was 0.5 , which implied the average between the minimum and maximum intensities of the field distribution. In the simulated pattern, black regions correspond to the non-patterned material, while white regions represent the patterned material. Along with the vertical standing wave $(\sim 193 \mathrm{~nm})$, larger period waves of $\sim 3.1,1.5$, and $0.8 \mu \mathrm{m}$ were obtained in the horizontal direction for $5^{\circ}, 10^{\circ}$, and $20^{\circ}$, respectively. The spacing of the surface (horizontal) grating decreased as the tilt angle approaches $0^{\circ}$. This was expected since the angle between the two interfering beams decreased. As the mirror tilt angle increased, the multilayer pattern diminished. Figure 2(c) illustrates the interference pattern arising from the superposition of the reflected waves as the transmission was varied from $0 \%$ to $40 \%$ with $10 \%$ increments, while constants were tilt angle $\left(5^{\circ}\right)$, thickness $(10 \mu \mathrm{m})$, and intensity $(0.5)$. As the transmission increased, the amount of intensity required increased to pattern the matrix. This represents a compromise between the absorption and the intensity. Figure 2(d) shows the interference pattern arising from the superposition of the reflected waves as the threshold intensity was varied from 0.5 to 0.8 with 0.1 increments, while constants were tilt angle $\left(5^{\circ}\right)$, transmission (20\%), and thickness $(10 \mu \mathrm{m})$. Figure 2(e) shows the interference pattern arising from the superposition of the reflected waves as the matrix thickness was varied from 2.0 to $10.0 \mu \mathrm{m}$ with $2.0 \mu \mathrm{m}$ increments, while constants were transmission $(20 \%)$, tilt angle $\left(5^{\circ}\right)$, and intensity threshold $(0.5)$. As the thickness of the matrix increased, the number of fringes in the multilayer structure also increased. At least, $5 \mu \mathrm{m}$ thick hydrogel was required to obtain $\sim 10$ stacks (Fig. 2(e)). Supplementary Figures S1-S5 show detailed simulation results. ${ }^{32}$ Thus, using simulation, we can predict the optical characteristics such as the periodicity of the transmission grating, the regions that will be patterned at a given absorption value, and the number of multilayer gratings that will be formed.

We have evaluated the optical properties of the gratings shown in Fig. 2(b). A finite element time domain allowed simulating the readout of the holograms. Figures 3(a)-3(c) show the simulations that describe normally propagated electric field of the incoming $\mathbf{1}$ and refracted $\mathbf{2}$ waves in transversal electric mode. The first order diffracted waves 3 were observed at $\sim 25^{\circ}, \sim 35^{\circ}$, and $\sim 65^{\circ}$ from the normal. 4 shows the second order of the diffraction. Enhanced online 


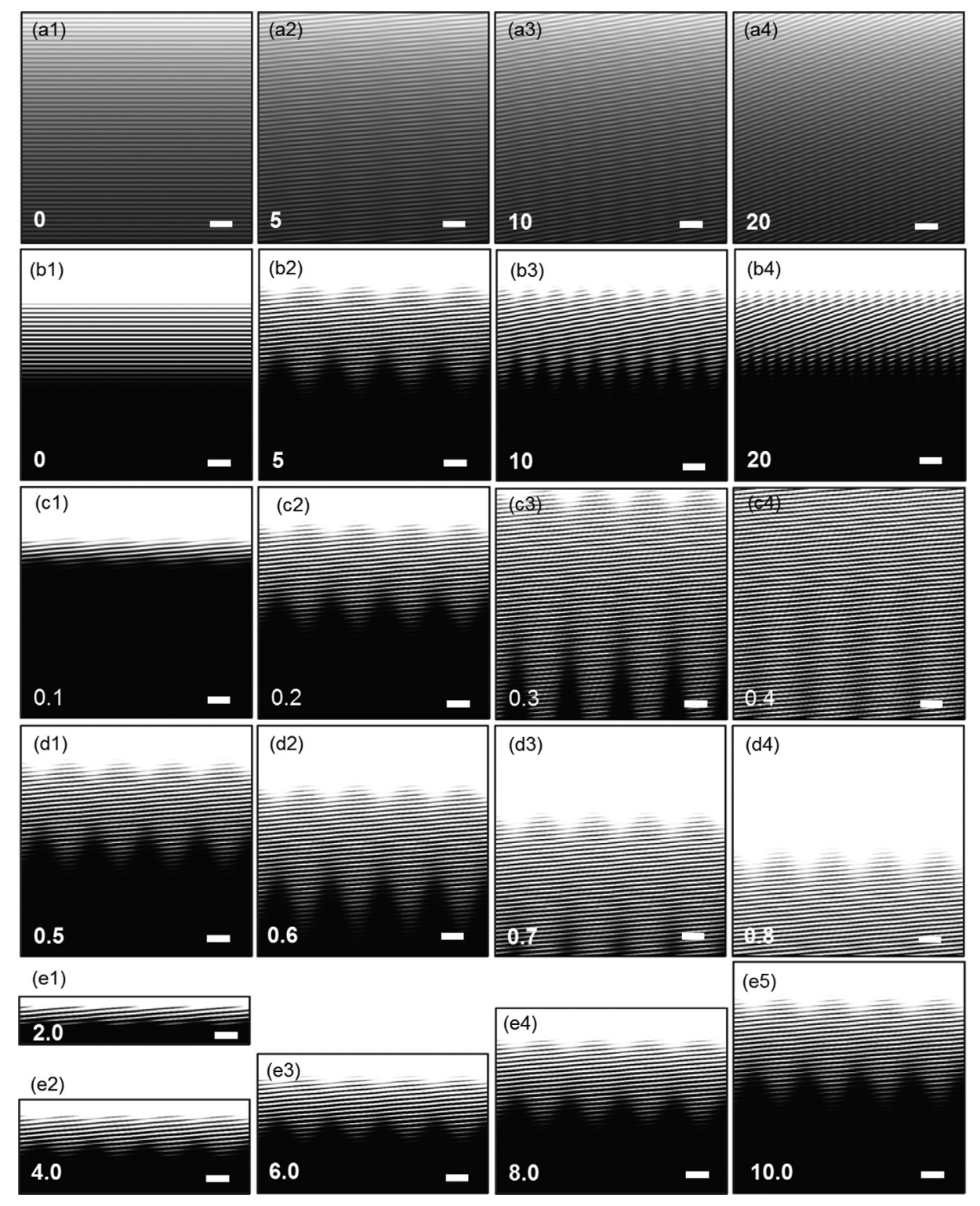

FIG. 2. Photochemical patterning based on the multi-beam interference in Denisyuk reflection mode. The incident wave is propagated from the top. (a) The intensity field distribution $\mathrm{I}=|\mathrm{E}|^{2}$ produced by laser light interference inside the matrix with tilt angles of $0^{\circ}-20^{\circ}$, created by three beams: (i) incident beam, (ii) beam reflected from the mirror, and (iii) beam reflected internally at the pHEMA-water environment interface. (b) Threshold of intensity of the cross section to represent patterned and nonpatterned regions as the tilt angle from the normal is varied from $0^{\circ}$ to $20^{\circ}$ with $5^{\circ}$ increments. The interference pattern arising from the superposition of the reflected waves as (c) the transmission is varied from 0.1 to 0.4 with 0.1 increments, (d) the intensity is varied from 0.5 to 0.8 , and (e) the polymer matrix thickness is varied from 2.0 to $10.0 \mu \mathrm{m}$ with $2.0 \mu \mathrm{m}$ increments. Scale bars $=1 \mu \mathrm{m}$.

multimedia movies 1-3 show the interaction of the electromagnetic waves with the photonic structures. ${ }^{32}$

The fabrication of holographic sensors consisted of the preparation of a polymer film, in situ nanoparticle growth, and formation of a hologram in the polymer matrix. The holograms were recorded in Denisyuk reflection mode
(Fig. 4(a)). The hologram was probed by reflection spectrophotometer (Fig. 4(b)). Supplementary material describes recording and probing of the holograms. ${ }^{32}$ Upon illumination at normal incidence using a supercontinuum white light laser, the photonic structure showed a narrow-band Bragg peak at $\sim 530 \mathrm{~nm}$ produced by the reflection grating. At the
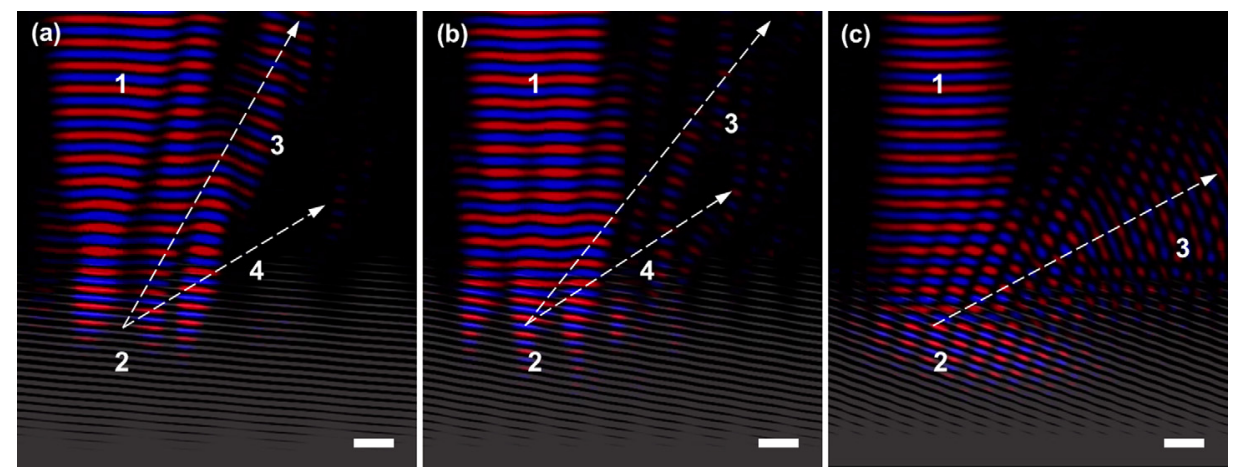

FIG. 3. Simulations of the optical readouts. A coherent source 1 of incident waves $(\lambda=532$ nm) is propagated onto the multilayer nanostructure recorded at (a) $5^{\circ}$, (b) $10^{\circ}$, and (c) $20^{\circ}$ slanted angles, refracting 2 and diffracting 3 light. 4 shows the second order diffraction. Scale bars $=1 \mu \mathrm{m}$. (Multimedia view) [URL: http://dx.doi.org/10.1063/1.4905352.1][URL: http://dx.doi.org/10.1063/1.4905352.2] [URL: http://dx.doi.org/10.1063/1.4905352.3] 
(a)

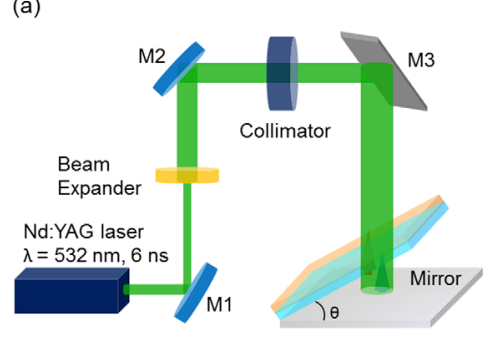

(d)

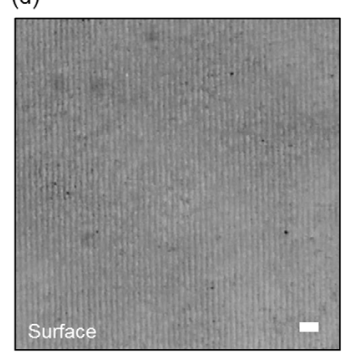

(e) (b)
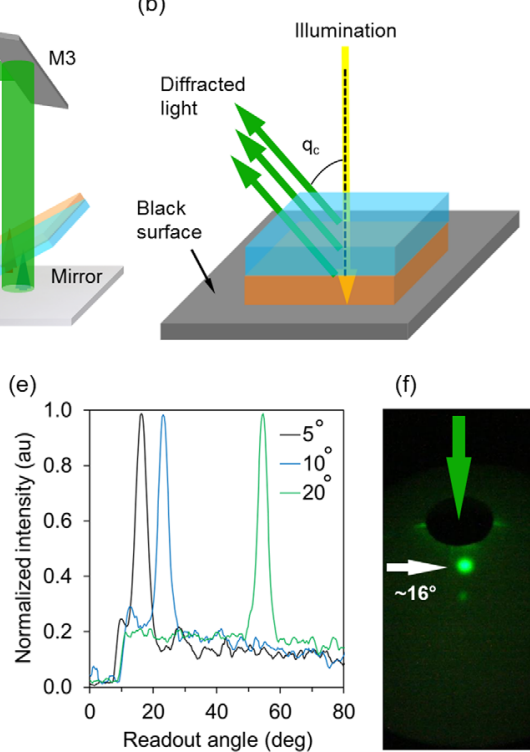

(c)

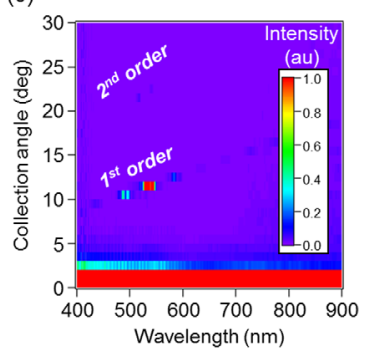

(h)

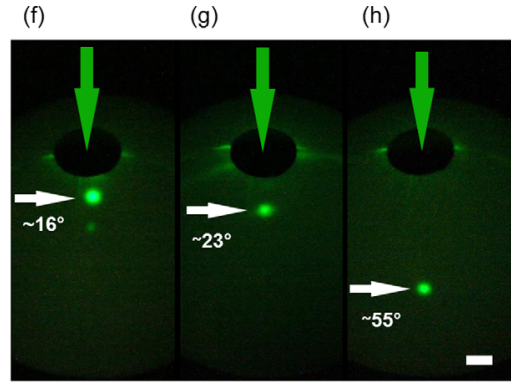

FIG. 4. Fabrication of a hologram in Denisyuk reflection mode and its optical characterization. (a) The laser light exiting the laser is deviated from a dielectric mirror (M1), expanded, deviated (M2), collimated and directed (M3) over the pHEMA matrix doped with Ag ${ }^{0}$ NPs. The reference and object beams form a standing wave, which photochemically patterns the recording medium. (b) Probing the hologram. (c) Angular-resolved measurements of the diffracted light from the hologram, showing a Bragg peak at $\sim 530 \mathrm{~nm}$. (d) The optical image of the transmission grating on the surface of the hologram fabricated at $5^{\circ}$ tilt angle. The period of the surface grating is $\sim 3 \mu \mathrm{m}$. Scale bar $=10 \mu \mathrm{m}$ (e) Angular measurements of the diffracted light from the holograms fabricated at $5^{\circ}$, $10^{\circ}$, and $20^{\circ}$. (f) Photographs of the variation in diffraction angles from $16^{\circ}$ to (g) $23^{\circ}$, and (h) $55^{\circ}$ shown in an integrating sphere. Scale bar $=5 \mathrm{~mm}$.

same time, a surface transmission grating creates higher orders of diffraction (Fig. 4(c)). The Bragg peak can be modulated from UV to near-infrared in sensing applications. As the target molecules bind to receptors in the hologram, the changes in Donnan osmotic pressure lead to structural or refractive index changes that alter the photonic band gap, where the diffracted light can be correlated with analyte concentrations from $\mu \mathrm{M}$ to $\mathrm{mM}$ range. ${ }^{14}$ Figure $4(\mathrm{~d})$ illustrates an optical image of the surface topography of the photonic structure in bright field showing a periodic grating. These transmission gratings are caused by the interference of the object beam and internally reflected light. The periodicity of the surface grating was measured as $\sim 3 \mu \mathrm{m}$ for a hologram recorded at $5^{\circ}$ tilt angle from the mirror plane, as predicted by the simulations. To support the results obtained from the simulations for different tilt angles (Fig. 3), holograms were recorded at $5^{\circ}, 10^{\circ}$, and $20^{\circ}$ tilt angles from the mirror plane. When a readout laser beam $(\lambda=532 \mathrm{~nm}$ and $1 \mathrm{~mW})$ normally illuminated the holograms, the gratings diffracted the light at $\sim 16^{\circ}, \sim 23^{\circ}$, and $\sim 55^{\circ}$ from the normal, respectively, (Fig. 4(e)). Figures 4(f)-4(h) show the photographs of the Bragg diffractions. The increase in the angle of the diffracted light might be attributed to the change in the refractive index of the hologram throughout the multilayer. Another explanation is that when the sample is tilted, the fringes inside the layer become more slanted due to the refraction at the air/recording material surface. The angles of the diffracted light from the normal were smaller than the angles predicted by the simulations; however, the increase of the diffraction angle with increasing recording tilt angle was consistent with the simulated model.

Kogelnik's coupled wave theory has explained the sinusoidal diffraction. ${ }^{30}$ Additionally, parallel stacked mirrors model showed a differential formulation of the Fresnel reflection within the grating. ${ }^{31}$ In the present work, light-matter interaction by high-energy pulsed laser recording does not follow the same principles. ${ }^{24,28}$ The reason is that the photochemical patterning described by the previous models implies that the change in the refractive index is proportional to the intensity of the recording beams producing a sinusoidal profile. However, the patterning of NPs is not linear with respect to the intensity of the recording beam in the present work. In contrast to the previous models, we consider that patterning of silver NPs occurs at a given threshold. This threshold in addition to the absorption required by the NPs limits the patterned regions within the recording medium.

We have studied hologram recording using a highenergy pulsed laser to evaluate the parameters that affect grating formation. A threshold should be passed to form a diffraction grating, hence rendering this image recording technique fundamentally different than photochemistry. Additionally, the grating should be produced at the lowest tilt angle to reduce the effect of the transmission grating in the readouts. We have also fabricated holograms with predictive optical properties at various diffraction angles using a pulsed laser, which produced multilayer Bragg and transmission gratings within pHEMA matrixes. However, a limitation of the high-energy pulsed laser recording is that it produces holograms with diffraction efficiencies less than 5\%, which needs to be improved for practical applications. The model and the fabrication technique can be applied to other holograms that are formed using complex objects such as concave and convex mirrors, prisms, and retroreflectors. Rationally designing diffraction gratings via high-energy pulsed laser recording will allow fabricating holographic patterns with predictive optical characteristics. 
${ }^{1}$ S. Tay, P. A. Blanche, R. Voorakaranam, A. V. Tunc, W. Lin, S. Rokutanda, T. Gu, D. Flores, P. Wang, G. Li, P. St Hilaire, J. Thomas, R. A. Norwood, M. Yamamoto, and N. Peyghambarian, Nature 451, 694 (2008).

${ }^{2}$ L. L. Huang, X. Z. Chen, H. Muhlenbernd, H. Zhang, S. M. Chen, B. F. Bai, Q. F. Tan, G. F. Jin, K. W. Cheah, C. W. Qiu, J. S. Li, T. Zentgraf, and S. Zhang, Nat. Commun. 4, 2808 (2013).

${ }^{3}$ Y. Montelongo, J. O. Tenorio-Pearl, C. Williams, S. Zhang, W. I. Milne, and T. D. Wilkinson, Proc. Natl. Acad. Sci. U. S. A. 111, 12679 (2014).

${ }^{4}$ Y. Montelongo, J. O. Tenorio-Pearl, W. I. Milne, and T. D. Wilkinson, Nano Lett. 14, 294 (2014).

${ }^{5}$ F.-K. Bruder, R. Hagen, T. Rölle, M.-S. Weiser, and T. Fäcke, Angew. Chem., Int. Ed. 50, 4552 (2011).

${ }^{6}$ S. Larouche, Y. J. Tsai, T. Tyler, N. M. Jokerst, and D. R. Smith, Nat. Mater. 11, 450 (2012).

${ }^{7}$ L. L. Brott, R. R. Naik, D. J. Pikas, S. M. Kirkpatrick, D. W. Tomlin, P. W. Whitlock, S. J. Clarson, and M. O. Stone, Nature 413, 291 (2001).

${ }^{8}$ S. Marchesini, S. Boutet, A. E. Sakdinawat, M. J. Bogan, S. Bajt, A. Barty, H. N. Chapman, M. Frank, S. P. Hau-Riege, A. Szoke, C. Cui, D. A. Shapiro, M. R. Howells, J. C. H. Spence, J. W. Shaevitz, J. Y. Lee, J. Hajdu, and M. M. Seibert, Nat. Photonics 2, 560 (2008).

${ }^{9}$ C. Trillo, A. F. Doval, S. Hernandez-Montes, X. L. Dean-Ben, J. C. Lopez-Vazquez, and J. L. Fernandez, Meas. Sci. Technol. 22, 025109 (2011).

${ }^{10}$ M. Hytch, F. Houdellier, F. Hue, and E. Snoeck, Nature 453, 1086 (2008).

${ }^{11}$ D. Gagnon, S. Lessard, M. Verhaegen, P. Mutchmore, P. Bouchard, F. R. Doucet, and M. Sabsabi, Appl. Opt. 51, B7 (2012).

${ }^{12}$ M. Antier, C. Larat, E. Lallier, J. Bourderionnet, J. Primot, and A. Brignon, Opt. Express 22, 23310 (2014).

${ }^{13}$ P. A. Blanche, A. Bablumian, R. Voorakaranam, C. Christenson, W. Lin, T. Gu, D. Flores, P. Wang, W. Y. Hsieh, M. Kathaperumal, B. Rachwal, O. Siddiqui, J. Thomas, R. A. Norwood, M. Yamamoto, and N. Peyghambarian, Nature 468, 80 (2010).

${ }^{14}$ A. K. Yetisen, I. Naydenova, F. C. Vasconcellos, J. Blyth, and C. R. Lowe, Chem. Rev. 114, 10654 (2014).

${ }^{15}$ A. K. Yetisen, M. M. Qasim, S. Nosheen, T. D. Wilkinson, and C. R. Lowe, J. Mater. Chem. C 2, 3569 (2014).

${ }^{16}$ A. K. Yetisen, Y. Montelongo, F. C. Vasconcellos, J. L. MartinezHurtado, S. Neupane, H. Butt, M. M. Qasim, J. Blyth, K. Burling, J. B.
Carmody, M. Evans, T. D. Wilkinson, L. T. Kubota, M. J. Monteiro, and C. R. Lowe, Nano Lett. 14, 3587 (2014).

${ }^{17}$ Y. Fuchs, O. Soppera, A. G. Mayes, and K. Haupt, Adv. Mater. 25, 566 (2013).

${ }^{18}$ L. R. Volpatti and A. K. Yetisen, Trends Biotechnol. 32, 347 (2014).

${ }^{19}$ N. M. Farandos, A. K. Yetisen, M. J. Monteiro, C. R. Lowe, and S. H. Yun, "Contact lens sensors in ocular diagnostics," Adv. Healthcare Mater. (published online 2014).

${ }^{20}$ A. K. Yetisen, J. L. Martinez-Hurtado, A. Garcia-Melendrez, F. C. Vasconcellos, and C. R. Lowe, Sens. Actuators, B 196, 156 (2014).

${ }^{21}$ Y. Fuchs, S. Kunath, O. Soppera, K. Haupt, and A. G. Mayes, Adv. Funct. Mater. 24, 688 (2014).

${ }^{22}$ C. P. Tsangarides, A. K. Yetisen, F. C. Vasconcellos, Y. Montelongo, M. M. Qasim, T. D. Wilkinson, C. R. Lowe, and H. Butt, RSC Adv. 4, 10454 (2014).

${ }^{23}$ A. K. Yetisen, H. Butt, F. C. Vasconcellos, Y. Montelongo, C. A. B. Davidson, J. Blyth, L. Chan, J. B. Carmody, S. Vignolini, U. Steiner, J. J. Baumberg, T. D. Wilkinson, and C. R. Lowe, Adv. Opt. Mater. 2, 250 (2014).

${ }^{24}$ J. L. Martinez-Hurtado, C. A. Davidson, J. Blyth, and C. R. Lowe, Langmuir 26, 15694 (2010).

${ }^{25}$ J. L. Martinez-Hurtado and C. R. Lowe, ACS Appl. Mater. Interfaces 6, 8903 (2014).

${ }^{26}$ V. N. Bagratashvili, A. O. Rybaltovsky, N. V. Minaev, P. S. Timashev, V. V. Firsov, and V. I. Yusupov, Laser Phys. Lett. 7, 401 (2010).

${ }^{27}$ A. O. Rybaltovskii, V. I. Gerasimova, N. V. Minaev, V. I. Sokolov, P. S. Timashev, E. A. Troitskaya, V. V. Firsov, V. I. Yusupov, and V. N. Bagratashvili, Nanotechnol. Russ. 5, 435 (2010).

${ }^{28}$ J. Chung, S. Han, D. Lee, S. Ahn, C. P. Grigoropoulos, J. Moon, and S. H. Ko, Opt. Eng. 52, 024302 (2013).

${ }^{29}$ F. C. Vasconcellos, A. K. Yetisen, Y. Montelongo, H. Butt, A. Grigore, C. A. B. Davidson, J. Blyth, M. J. Monteiro, T. D. Wilkinson, and C. R. Lowe, ACS Photonics 1, 489 (2014).

${ }^{30}$ H. Kogelnik, Bell Syst. Tech. J. 48, 2909 (1969).

${ }^{31}$ D. Brotherton-Ratcliffe, Appl. Opt. 51, 7188 (2012).

${ }^{32}$ See supplementary material at http://dx.doi.org/10.1063/1.4905352 for the diffraction simulation movies, the model for superpositioning of waves and thresholding, synthesis of the recording medium, recording the holograms in denisyuk reflection mode, hologram probing, and detailed simulation results. 